\title{
Ionization and Dissociation of the Formic Acid Molecule by Protons and Electrons
}

\author{
S. Pilling ${ }^{1,2}$, A. C. F. Santos ${ }^{3}$, H. M. Boechat-Roberty ${ }^{1}$, G. G. B. de Souza ${ }^{2}$, \\ M. M. Sant' Anna $^{3}$, A. L. F. Barros ${ }^{3}$, W. Wolff ${ }^{3}$, and N. V. de Castro Faria ${ }^{3}$ \\ ${ }^{1}$ Observatório do Valongo, Universidade Federal do Rio de Janeiro, \\ Ladeira Pedro Antônio 43, CEP 20080-090, Rio de Janeiro, RJ, Brazil \\ ${ }^{2}$ Instituto de Química, Universidade Federal do Rio de Janeiro, \\ Ilha do Fundão, CEP 21949-900, Rio de Janeiro, RJ, Brazil \\ 3 Instituto de Física, Universidade Federal do Rio de Janeiro, \\ Caixa Postal 68528, CEP 21941-972, Rio de Janeiro, RJ, Brazil
}

Received on 29 July, 2005

\begin{abstract}
Ionization and dissociation of the formic acid molecule $(\mathrm{HCOOH})$ by energetic charged particles have been studied for $1 \mathrm{keV}$ electrons and nearly equivelocity $2 \mathrm{MeV}$ protons. Whereas the fragmentation pattern induced by impact of electron and proton show close similarities, some distinctions in the fragmentation yields were observed for these equivelocity projectiles.
\end{abstract}

Keywords: Molecular fragmentation; Ionization

\section{INTRODUCTION}

The solar wind (or stellar winds) consists of energetic charged particles like electrons, protons, alpha particles (helium nucleus) that interact with planetary atmospheres and comets, producing ionization and dissociation of the molecules present in such objects. Ionization and dissociation of the formic acid molecule by fast ions and electrons have been the subject of much study due to its fundamental importance in determining the ionization, thermal balance and chemistry in interstellar clouds, planetary atmospheres and comet tails $[1,2]$. The formic acid molecule $(\mathrm{HCOOH})$ has been observed in several astronomical sources such as protostellar ices NGC 7538:IRS9, condritic meteorites [3], and dark molecular clouds $[4,5]$ and comets $[6,7]$. The destruction of the formic acid molecule by soft X-rays, present in star forming regions, has recently been studied in laboratory conditions with synchrotron radiation [8].

Recently, differences in the single and multiple-ionization cross sections of atoms by equal velocity electrons and protons were reported for targets with high atomic number [9]. Additionally, differences were also encountered in the multiple-ionization cross sections of atoms by positrons and electrons [10-12]. In the case of complex molecular targets, the pioneering study of ionization and fragmentation of molecules of biological relevance induced by either protons or electron was performed by Coupier et al. [13]. It has been observed that protons and electrons lead to the same fragmentation patterns.

In the following we present experimental results on the ionization and fragmentation of the formic acid molecule upon interaction with fast electrons and protons.

\section{EXPERIMENTAL SETUP}

\section{A. Proton impact}

A tightly collimated, $2.0 \mathrm{MeV}$ proton beam is delivered by the 1.7 MV Tandem accelerator of the Federal University of Rio de Janeiro. The beam is charge analyzed by an electric field placed just before crossing at right angles an effusive jet of formic acid molecules, in order to improve its charge state purity, since the proton beam can become partially neutralized due to the interactions with the residual gases present in the beam line. The emergent beam is recorded by a channeltron detector housed in a detection chamber downstream of the gas cell, after charge state analysis by a second electric field, so that it can be separated from the $\mathrm{H}^{0}$ beam originated from single electron capture in interactions with the gas target.

In the projectile velocity range studied in this paper, the direct ionization process (the charge of the projectile does not change during the collision) is the dominant collision channel producing target ionization. Formic acid, that has been degassed prior to the experiment in freezing, pumping and thawing cycles, is introduced in vapor form, through a needle, into the interaction region. The atomic and molecular ionic fragments, produced by the incident beam under single collision regime, are accelerated by a two-stage electric field and detected by another channeltron detector.

The time-of flight (TOF) measurement, running in the coincidence mode, is then started by the detection of the proton beam and stopped by ions extracted onto the TOF-detector. The first stage of the electric field is produced by a plate-grid system and the primary beam passes through its middle with a $130 \mathrm{~V} / \mathrm{cm}$ electric field. The extracting electric field was raised up to to $950 \mathrm{~V} / \mathrm{cm}$ in order to assure complete recoil ions collection, however, no difference was observed in the recoils intensity. The mass resolution of the time-of-flight spectrometer is not high enough to allow the discrimination of some measured fragments from their neighboring ions of a 


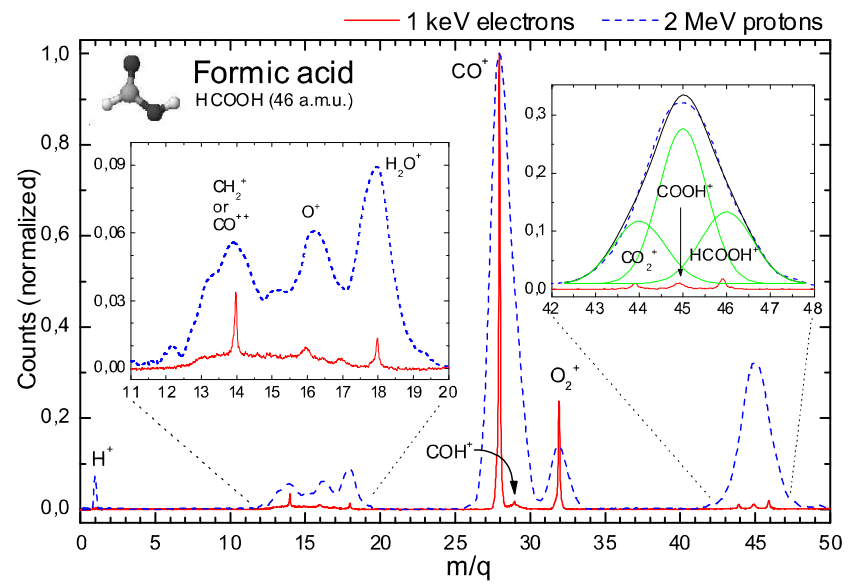

FIG. 1: Mass spectra of formic acid fragments by $2 \mathrm{MeV}$ protons (dashed line) and $1 \mathrm{keV}$ electrons (full line).

mass-to-charge ratio differing by only 1 a.m.u. (see Figure 1).

\section{B. Electron impact}

The apparatus used for the electron impact experiment was described previously in detail [14]. In brief, a non-pulsed $1 \mathrm{keV}$ electron beam crossed perpendicularly the gaseous formic acid target. The atomic and molecular fragments produced by the incident beam, under single collision conditions, are accelerated by a two-stage electric field and detected by a microchannel plate detector mounted in a chevron configuration. This detector provides stop signals to the time-to-digital converter started by the signals from the secondary low energy electrons accelerated in the opposite direction. The first stage of the electric field consists of a plate-grid system with the primary beam passing through its middle with a $60 \mathrm{~V} / \mathrm{cm}$ electric field. Ions with energies up to $5 \mathrm{eV}$ have $100 \%$ collection efficiency.

\section{RESULTS}

Figure 1 shows the time-of-flight spectra, expressed in terms of mass over charge ratio, for the fragmentation of formic acid by $2.0 \mathrm{MeV}$ protons and $1 \mathrm{keV}$ electron impact. This figure gives an illustration of the distinct mass resolutions of the two coincidence setups, showing a clear separation of the $\mathrm{COH}^{+}$or $\left(\mathrm{HCO}^{+}\right)$and $\mathrm{CO}^{+}$peaks in the electron impact case, but not in the proton impact case. In the inset Fig. 1 at right, we can see a deconvolution process of highest mass peak obtained in the proton case showing the contributions of fragments $\mathrm{CO}_{2}^{+}, \mathrm{COOH}^{+}$and $\mathrm{HCOOH}^{+}$superimposed with the those peaks obtained in the electron case.

Generally speaking, both projectile interactions lead to the same formic acid fragments, but the overall relative intensities of the fragments are clearly distinct for each experiment. The different mass resolution in the experimental setups may con-

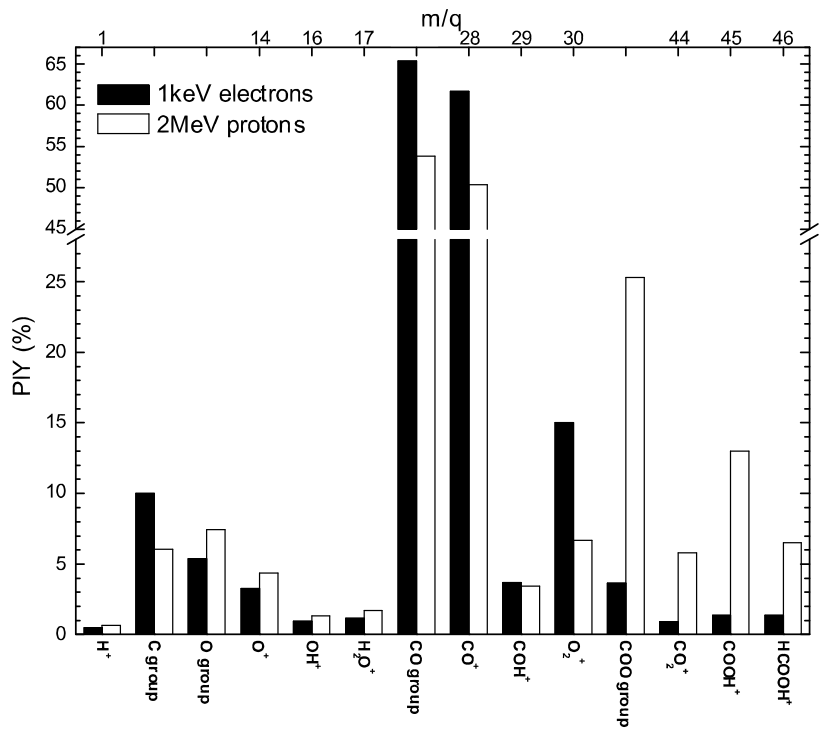

FIG. 2: Partial ion yield (PIY) of formic acid molecule by $1 \mathrm{keV}$ electron (filled bars) and $2 \mathrm{MeV}$ proton impact.

tribute to the exhibition of slightly different peak widths for a specific ionic fragment. In addition, a comparison between both mass spectra conveys some interesting observations. For both projectiles, the $\mathrm{CO}^{+}$fragment dominates the spectra. In the proton impact case, this peak is clearly convoluted with the $\mathrm{COH}^{+}$or $\left(\mathrm{HCO}^{+}\right)$peak. Another interesting observation concerns the relative intensity of the $\mathrm{H}^{+}$. In the proton case, it is roughly three times higher than in the electron impact case. Whether this is a result of the spectrometer discrimination against high energy fragments (due to its low mass, $\mathrm{H}^{+}$ takes most of the kinetic energy available in the dissociation process) or associated with different breakup process is uncertain. On the other hand, the relative intensity of the $\mathrm{O}_{2}^{+}$ peak in the electron impact case overcomes by a factor 2 the corresponding peak in the proton impact case.

The water peak (18 a.m.u.) represents an intrinsic recombination of some dissociation products of $\mathrm{HCOOH}$ also reported in the literature [8]. The base pressure in the vacuum chamber was in the $10^{-8}$ Torr range and during the experiment the chamber pressure was maintained below $10^{-5}$ Torr. The pressure at the interaction region (volume defined by the gas beam and the proton beam intersection, about 1-3 $\mathrm{mm}^{3}$ ) was estimated to be $\sim 1$ Torr $\left(10^{16}\right.$ mols $\left.\mathrm{cm}^{-} 3\right)$. The measurements were made at room temperature.

Figure 2 shows the partial ion yield (PIY) of the formic acid molecule for $1 \mathrm{keV}$ electron and $2 \mathrm{MeV}$ proton impact as a function of the mass-to-charge ratio of the fragments. The PIY value for each fragment $i$ was obtained by

$$
P I Y_{i}=\left(\frac{A_{i}}{A_{t}^{+}} \pm \frac{\sqrt{A_{i}}+A_{i} \times E R / 100}{A_{t}^{+}}\right) \times 100 \%
$$

were $A_{i}$ is the area of a fragment peak, $A_{t}^{+}$is the total area of the PEPICO spectrum. $E R=2 \%$ (in the electron impact) and $10 \%$ (in the proton impact) is the estimated error factor for 
the data treatments.

Since the experiments have presented different mass resolution we have considered some fragments together in groups (ex. $\mathrm{CO}$ group: $\mathrm{CO}^{+}+\mathrm{COH}^{+}$) to avoid uncertainties in the yield comparison. The fragments from each group are indicated by the upper arrows. The most expressive differences were found in the yields of $\mathrm{COO}$ group $\left(\mathrm{CO}_{2}^{+}+\mathrm{COOH}^{+}\right.$ $+\mathrm{HCOOH}^{+}$) and $\mathrm{O}_{2}^{+}$. The former has presented an yield 4 times higher in the $2 \mathrm{MeV}$ protons experiment and the latter about 2 times higher in the experiment with $1 \mathrm{keV}$ electron.

\section{CONCLUSIONS}

The present work compares the ionization and fragmentation of the formic acid molecule by $2.0 \mathrm{MeV}$ protons and by roughly equivelocity $1 \mathrm{keV}$ electron impact. Despite the fact that both projectiles lead to the same fragmentation pattern, we observed differences in the relative intensities of the fragments. The $1 \mathrm{keV}$ electrons lead to a stronger fragmentation of the formic acid molecules than the equivelocity $2.0 \mathrm{MeV}$ protons. This is in direct accordance with the corresponding differences observed in the atomic case, and has been suggested to arise from projectile charge effects. This paper alerts about the errors which can arise if one uses single-ionization cross sections by electron impact to normalize proton and positron cross sections at high velocities.

\section{Acknowledgments}

The authors would like to express their gratitude to the Brazilian funding agencies FUJB (UFRJ), CAPES, CNPq, and FAPERJ.
[1] C. J. Latimer, Adv. Atom. Mol. Opt. Phys. 30, 105 (1993).

[2] A. C. F. Santos, W. S. Melo, M. M. Sant'Anna, G. M. Sigaud, and E. C. Montenegro, Braz. J. Phys (this issue).

[3] J. F. Briscoe and C. B. Moore, Meteoritics 28, 330 (1993).

[4] P. Ehrenfreund and W. A. Schutte, Ad. Space Res. 25, 2177 (2000).

[5] M. Ohishi, W. M. Irvine, and N. Kaifu, (1992) Astrochemistry of Cosmic Phenomena: edited by P. D. Singh, Kluwer Academic Publishers, Dordrecht.

[6] D. Bockelúe-Morvan, D. C. Lis, J. E. Wink, D. Despois, J. Crovisier, R. Bachiller, D. J. Benford, N. Biver, P. Colom, J. K. Davies, et al., Astronomy and Astrophysics 353, 1101 (2000).

[7] N. Biver, D. Bockelúe-Morvan, J. Boisser, P. Colom, J. Crovisier et al., IAU Symp. 291 - Asteroids, Comets and Meteories P5.1, 43 (2005).

[8] H. M. Boechat-Roberty, S. Pilling, and A. C. F. Santos, Astronomy and Astrophysics 438, 915 (2005).
[9] W. S. Melo, A. C. F. Santos, M. M. Sant'Anna, G. M. Sigaud, and E. C. Montenegro, J. Phys. B: At. Mol. Opt. Phys. 35 L187 (2002).

[10] A. C. F. Santos, A. Hasan, T. Yates, and R. D. DuBois, Phys. Rev. A 67, 052708 (2003).

[11] A. C. F. Santos, A. Hasan, and R. D. DuBois, Phys. Rev A 69, 032706 (2004).

[12] A. C. F. Santos, A. Hasan, and R. D. DuBois, Phys. Rev. A 71, 034701 (2005).

[13] B. Coupier, B. Farizon, M. Farizon, M. J. Gaillard, F. Gobet, N. V. de Castro Faria, G. Jalbert, S. Ouaskit, M. Carrú, B. Gstir, G. Hanel, S. Denifl, L. Feketeova, P. Scheier, and T. D. Mrk, Eur. Phys. J. D 20, 459 (2002).

[14] J. B. Maciel, E. Morikawa, and G. G. B. de Souza, Syc. Rad. Instr. AIP Conference Proceedings 417, 22 (1997). 